\title{
Perspective
}

PERSPECTIVE Actualité en histoire de l'art

1 | 2021

Portugal et espaces lusophones

\section{Images postcoloniales : de la révolution des Eillets aux processus d'indépendance. Ouvrages de propagande, de résistance et de liberté (1974-1984)}

Postcolonial Images: From the Carnation Revolution to the Independence

Processes. Publications for Propaganda, Resistance and Freedom (1974-1984)

Postkoloniale Bilder: Von der Nelkenrevolution auf den Weg zur Unabhängigkeit.

Propaganda, Widerstand und Freiheit im Buch (1974-1984)

Imagens pós-coloniais: da revolução de Abril aos processos de independência.

Livros de propaganda, resistência e liberdade (1974-1984)

Immagini postcoloniali: dalla Rivoluzione dei garofani ai processi di

indipendenza. Opere di propaganda, di resistenza e di libertà (1974-1984)

\section{Susana Lourenço Marques}

Traducteur : Thomas Resendes

\section{(2) OpenEdition}

Journals

\section{Édition électronique}

URL : https://journals.openedition.org/perspective/24249

DOI : $10.4000 /$ perspective. 24249

ISSN : 2269-7721

Éditeur

Institut national d'histoire de l'art

\section{Édition imprimée}

Date de publication : 14 octobre 2021

Pagination : 231-246

ISBN : 978-2-917902-91-2

ISSN : $1777-7852$

Référence électronique

Susana Lourenço Marques, «Images postcoloniales : de la révolution des Eillets aux processus d'indépendance. Ouvrages de propagande, de résistance et de liberté (1974-1984) », Perspective [En ligne], 1 | 2021, mis en ligne le 31 décembre 2021, consulté le 13 mai 2022. URL : http:// journals.openedition.org/perspective/24249; DOI : https://doi.org/10.4000/perspective.24249 


\title{
Images postcoloniales : de la révolution des OEillets aux processus d'indépendance
}

\author{
Ouvrages de propagande, de résistance \\ et de liberté (1974-1984)
}

Susana Lourenço Marques

ODETTE - Oui, d'accord, mais comment il sort ? Comment il s'en sort le Portugal? Comment il va de l'entrée à la sortie ? On peut, au moins, se demander ça, comment ça va de l'entrée à la sortie de la machine, puisque notre pouvoir de journalisme, il est là, merde ! Il n'est pas ailleurs, il n'est ni en France ni au Portugal mais entre les deux ${ }^{1}$.

\section{Photographie, appropriation et histoire}

Relisant aujourd'hui la déclaration de Roland Barthes selon laquelle « Le même siècle a inventé l'histoire et la photographie ", c'est le caractère indissociable de nos conceptions actuelles de l'histoire et de la photographie qui semble finalement, et paradoxalement, décisif. Grâce au découpage, au cadrage, à la profondeur et à la possibilité qu'elle offre de différer, la photographie prend part à l'ère des " révolutions, des contestations, des attentats, des explosions, bref des impatiences ${ }^{2}$ ". Leurs marches distinctes se transforment alors en une durée commune, selon une juxtaposition des chronologies, un montage du temps et de l'événement lui-même. Comme l'ont revendiqué Sigfried Kracauer, Marc Ferro, Jacques Rancière ou Georges Didi-Huberman, l'intégration de la photographie et du cinéma dans l'élaboration de l'histoire du XXe siècle s'est avérée être l'outil essentiel d'un changement de paradigme du travail de l'historien. Pour Marc Ferro, il s'agit d'une adhésion à la présence de l'image, à la façon dont celle-ci survole l'histoire, en examine les détails, les marques et contingences du temps. L'historien doit penser à partir d'un moyen de production et de transmission visuelle : il se met à " lire et à écouter les images ${ }^{3}$ ", conscient de l'instrumentalisation qui s'y profile.

La photographie et le cinéma permettent de sonder les mouvements du temps historique et confèrent au document une temporalité inédite. Comme l'affirme Jean-Luc Godard, rendre visible le montage, c'est matérialiser l'histoire comme une épopée autobiographique visuelle. C'est un travail d'archéologie des mouvements sociaux, politiques et culturels à travers les images du cinéma : "Apprendre à voir, pas à lire ${ }^{4}$. " 
Dans le film Comment ça va ? (1976), Jean-Luc Godard et Anne-Marie Miéville réalisent un film documentaire à partir d'une photographie publiée dans Libération, sur l' «été chaud " de 1975 au Portugal. Ce documentaire reflète l'ambiguïté des images médiatiques, leur incessante appropriation et la façon dont elles façonnent et brouillent notre lecture de la révolution. Dans le film, la photographie représente la révolution dans sa dimension cyclique, comme un mouvement de transformation historique et politique qui renforce la véridicité des images et les manifestations de pouvoir qui s'y déploient.

Comment ça va? est un essai sur la façon dont le cinéma et la photographie reflètent les stratégies de diffusion de la révolution, ainsi que ses contradictions et dénaturations ultérieures. Comme le souligne Gilles Deleuze, dans ce film, la relation entre les images est une question d'arythmie, de dissonance, qui s'écarte du concept d'auteur pour progresser vers ce qu'il nomme " la vision indirecte libre ". Godard et Miéville s'intéressent à l'émergence des images dans les interstices de la révolution. En examinant la façon dont se dilate leur processus de manipulation - sur le plan de la production, de l'archivage et de la réception, ils revendiquent une pédagogie critique qui leur est indispensable.

\section{Image, propagande et (dé)colonisation}

Au cours des quarante-huit années de dictature, la photographie et le cinéma ont été deux outils essentiels de la diffusion idéologique de l'Estado novo, notamment grâce à la mise en œuvre de la " politique de l'esprit " ", le programme culturel créé par António Ferro afin de définir les orientations esthétiques et le modèle de propagande culturelle du régime. Transformés en outils d'exaltation impérialiste, la photographie et le cinéma ont été utilisés dans des mises en scènes apologétiques démontrant l'ambition et la modernité des réformes politique, économique, culturelle et technologique du pays et de ses territoires ultramarins.

Les politiques répressives menées contre la création et les procédés de légitimation artistique mis en ouvre pendant la dictature - comprenant des logiques de censure et divers mécanismes de surveillance et d'interdiction -, ont été contrebalancés par un solide programme de contrôle et de réglementation du secteur de l'édition, entre autres à travers la création d'une industrie d'imprimerie portugaise, reconnue comme vecteur de propagande et instrument de création patriotique. Dans un contexte de fort analphabétisme de la population portugaise, l'Agence générale des colonies et le Secrétariat national de la propagande (SPN) étaient chargés de propager les succès de la mission coloniale auprès du peuple portugais et des puissances européennes.

La dynamisation du secteur de la presse illustrée, soutenue par l'édition d'albums et de monographies ethnographiques, étaient indispensables pour garantir la circulation et l'accès aux informations orchestrées par le régime, preuves de l'intention militante, délibérée et méthodique de diffuser une idéologie capable de reproduire de façon exemplaire l'hégémonie nationale 7 . Suite à la publication de l'ouvrage emblématique Portugal 1934 et de l'étude Arte Indígena Portuguesa (Ática, 1934) du sculpteur Diogo de Macedo et du poète Luís de Montalvôr, l' " Exposiçào Colonial Portuguesa " est organisée au Portugal, à Porto, pour la première fois, sous la direction de Henrique Galvão. Cette manifestation donna lieu, entre autres, à la publication d'un album photographique éponyme, signé par Domingos Alvão, ainsi qu'à celle d'Etnografia Angolana (subsídios) de Fernando Mouta. L'« Exposição Histórica da Ocupação », présentée en 1937 au pavillon du Parque Eduardo VII de Lisbonne, entérine cette recherche et rend hommage à la tradition expansionniste du Portugal, en démontrant que « La Nation continue d'exercer son activité en outre-mer, selon des principes fidèles à sa tradition humanitaire et colonisatrice ${ }^{8}$. " En 1938, à l'occasion de l'Expo-Feira Angola et de la création de la Missão cinegráfica às 
colónias de África ( "mission cinématographique dans les colonies d’Afrique ») dirigée par Carlos Selvagem, la documentation et la propagande du régime s'étendent hors du territoire continental. La stratégie géopolitique portugaise se consolide dans les territoires d'outre-mer, dans le but de renforcer durablement la souveraineté du régime, suite au revers de l'ultimatum britannique de 1890.

D'après Eduardo Lourenço, le « colonialisme innocent ${ }^{10}$ " semble se situer entre la mentalité colonialiste organique et structurelle dérivée d'un comportement historique et une forme de rejet, voire d'imperceptibilité de cette même structure - du système colonial lui-même. Ainsi, la prolifération des images coloniales, portées notamment par le cinéma et la photographie, s'est détachée d'une réalité sociale documentée pour développer à la place une idéalisation de la vie quotidienne des territoires d'outre-mer et façonner l'image de ceux qui y sont représentés.

C'est en opposition à ce processus lent et brutal de soumission politique et sociale que les mouvements armés de libération nationale ont commencé à s'affirmer à partir des années 1950, en Guinée Bissau (avec le PAIGC) à l'initiative d'Amílcar Cabral, en Angola (avec le Mouvement populaire de libération de l'Angola ou MPLA, l'Union nationale pour l'indépendance totale de l'Angola ou UNITA et l'Union des populations de l'Angola, ou UPA) et plus tard au Mozambique (avec le FRELIMO), donnant lieu à treize années de guerre coloniale qui aboutissent à une transition démocratique au Portugal, puis à un processus de décolonisation laborieux et prolongé, mené par le Mouvement des forces armées entre 1974 et 1975.

La fin de la politique impérialiste de l'Estado novo, puis la déclaration d'indépendance des territoires dits d'outre-mer, s'inscrivent dans l'imaginaire d'une lutte anti-impérialiste plus large. Comme l'a analysé Nadine Siegert :

Sur le continent africain, cette pratique de décolonisation culturelle - basée sur les écrits séminaux de penseurs tels que Frantz Fanon ou Amílcar Cabral - s'est manifestée, sous des formes visuelles pendant les périodes suivant les indépendances, comme une partie intégrante du processus de conceptualisation de la culture nationale et de l'agitation politique ${ }^{11}$.

C'est donc dans le négatif des images coloniales que les images postcoloniales trouvent leur essor - images photographiques et cinématographiques réalisées au cour d'un processus de résistance, de protestation et d'expression de la liberté. En revendiquant leur opposition et leur dénonciation, ces images permettent, comme le souligne James R. Ryan, « de servir une quantité de buts inédits, avec notamment la construction d'une Persona, de nouvelles appartenances de classe et des nationalismes postcoloniaux ${ }^{12}$ ». La publication de ces images sous la forme d'ouvrages monographiques, dans les premières années qui ont suivi l'indépendance, a permis de renforcer la persévérance contre l'oubli, tout en façonnant une nouvelle identité située entre l'héritage du passé colonial et ce qu'Achille Mbembe a nommé « le vieil imaginaire de la révolution et de l'anticolonialisme, la vieille thématique anti-impérialiste, aux thèses nativistes ${ }^{13}{ }^{\prime}$.

\section{Livres et photographies : protestation, résistance et liberté (1974-1984)}

Dès 1974, on assiste à une intense production de publications et d'expositions d'images mettant en avant la rupture et les tensions avec le régime dictatorial et le passé colonial. On observe notamment une transformation progressive des modèles de circulation et de légitimation, tant sur le plan des présupposés esthétiques et du propos auctorial que sur 
celui des formats d'expositions et d'éditions, en parfaite cohérence avec la révision des conditions de production artistique et l'ouverture à l'expérimentation et à la recherche qui se déploie dans leur interaction.

La fin de la censure et l'accès de la population à un champ de textes et d'images auparavant interdits sont deux facteurs décisifs du changement de stratégie et de l'autonomie des pratiques éditoriales au Portugal. Plus précisément, les livres illustrés constituent une iconographie active de la protestation et de la résistance. Ils illustrent l'enthousiasme croissant et caractéristique de cette période au cours de laquelle, selon l'étude de Flamarion Maués Silva, soixante-sept maisons d'éditions spécialisés dans les ouvrages à caractère politique ont été créées au Portugal : " quarante-huit d'entre elles pouvant être qualifiées de maisons d'édition de la révolution, leur apparition et leur activité coïncidant avec le processus révolutionnaire de $1974^{14}$ ". À l'intérieur de cet univers figurent des publications anarco-satiriques comme O livrinho vermelho do galo de Barcelos (1975) ou Os Salazarentos (1975), mais aussi des essais photo-littéraires comme O Último Dia da Pide (1974) du Movimento democrático do Porto (avec des photographies d'António Amorim, des poèmes d'Orlando da Costa, Fernando Assis Pacheco, Luísa Ducla Soares et des témoignages d'ex-prisonniers politiques), ou encore Uma Certa Maneira de Cantar (avec un reportage photographique de Costa Martins sur la réforme agraire et des poèmes de José Gomes Ferreira et Ary dos Santos), qui forgent et véhiculent de diverses manières le désir d'émancipation et la fin d'une longue période de subordination.

De façon moins constante mais tout aussi vigoureuse, il faut noter l'activité éditoriale menée par les mouvements indépendantistes en Angola ou au Mozambique, aux objectifs de propagande évidents, à laquelle s'ajoute le point de vue de photoreporters indépendants qui ont documenté le processus de décolonisation, au service des agences de presse internationale. Des ouvrages tels que With Freedom in their Eyes (1976), un reportage photographique du cinéaste et photographe américain Robert Kramer qui recoupe son film Scenes from the Class Struggle in Portugal (1977), réalisé au lendemain de la révolution des Eillets; Bilder aus Angola (1979) de la photographe allemande Jochen Moller ; Landet är vårt, broder: en bok om Angola (1979) du photographe suédois Sven Åsberg; ou Woods, Spirits and Warriors (1976) du photographe japonais Tadahiro Ogawa sont autant de témoignages caractéristiques du contexte social et politique de ces pays, des processus de transition des pouvoirs, mais aussi de l'intérêt qu'ils ont suscité et de leur influence à l'échelle internationale.

Malgré la distance et leur faible rayonnement géographique, ces publications permettent la mise en adéquation du concept de não inscrição ( "non-inscription »), forgé par des auteurs comme José Gil ou Eduardo Lourenço, avec une anesthésie, un oubli ou une amputation de l'imaginaire impérial. Selon Elsa Peralta, " Le Portugal s'imagine encore comme un Empire. La guerre et l'idée d'un retour se poursuivent à l'intérieur de cet imaginaire sans possibilité d'inscription ${ }^{15}$ ", comme le démontre le consensus visuel qui a marqué le processus de décolonisation, tant au Portugal qu'en Angola ou au Mozambique.

L'intégration récente d'une partie de ces publications dans des expositions, ainsi que la publication de certaines anthologies des meilleurs livres photographiques du $\mathrm{XX}^{\mathrm{e}}$ siècle - c'est le cas de The Photobook: a History Volume (2014) de Martin Parr et Gerry Badger; de l'exposition «Photobook Phenomenon » (2017); ou du projet Africa in the Photobook (2015) de Ben Krewinkel -, pose un second problème quant à leur nature, leur réactivation et leur revalorisation historique. En leur attribuant une valeur cultuelle fondamentalement opposée - le phénomène et l'exclusivité de la collection -, la muséification de ces publications semble avoir pour conséquence un anéantissement de leur dimension politique et la décontextualisation de leur environnement social d'origine. 
$\mathrm{Au}$ sein de cet univers de publications, nous distinguons une sélection spécifique d'ouvrages, analysés ci-après, qui mettent en évidence la coexistence d'un espace de contre-culture émergente et la manière dont les images gravent, de façon inédite, un désir de liberté, un geste de résistance et la condition de leur rupture avec le régime.

\section{Du Portugal à l'Angola : documenter les événements}

Portugal Livre (fig. la-b) propose une vision kaléidoscopique des événements de la semaine du 25 avril au $1^{\text {er }}$ mai 1974, grâce à un montage photographique retraçant les mouvements des forces armées, les scènes de liesse et les manifestations urbaines de la population, la libération des prisonniers politiques, le retour des exilés politiques et la chute des symboles du régime. Publié en juin 1974 par la maison d'édition Editorial O Século, suite à l'exposition éponyme

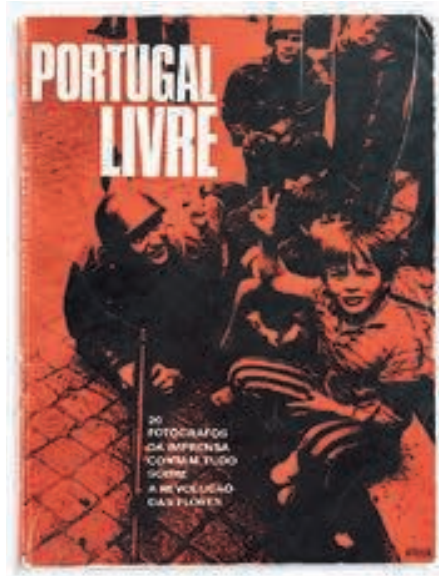

1a-b. Portugal livre (première de couverture et double page 18-19), Lisbonne, Editorial O Século, 1974. qui s'était déroulée au Palácio Foz, à Lisbonne, cet ouvrage rassemble centre trente photographies de vingt photographes différents - dont Alfredo Cunha, Carlos Gil, Eduardo Gageiro, Inácio Ludgero et Teresa Montserrat, avec une proposition graphique audacieuse coordonnée par Luis Filipe da Conceição et Vitorino Martins.

La variation des plans et des points de vue est amplifiée par un certain agencement des images dans la page et leur recadrage particulier. Ce procédé révèle l'urgence de ses conditions de réalisation et démontre en même temps la composante humaniste qui s'exprime désormais. Comme le décrit Fernando Assis Pacheco dans son introduction,

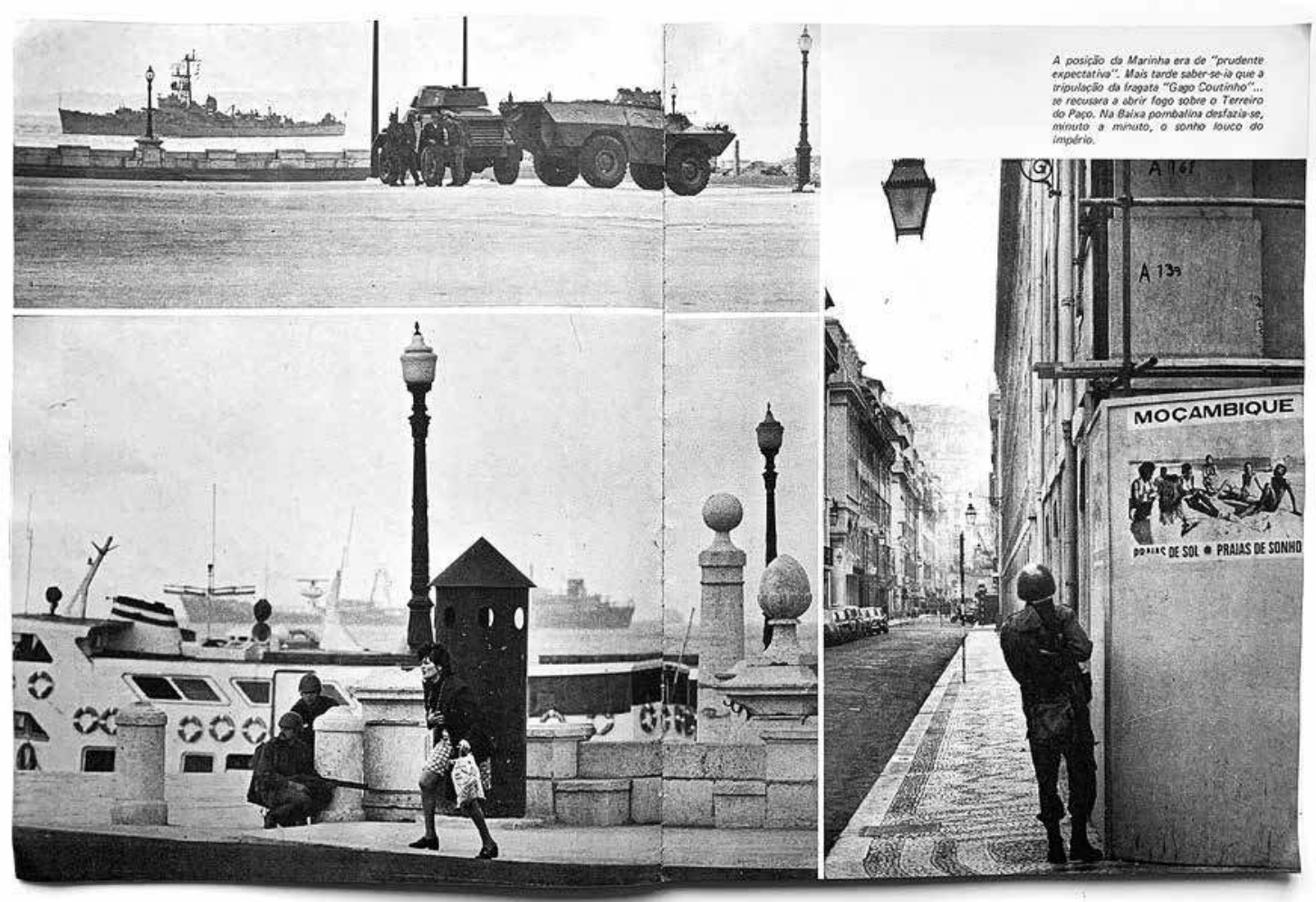


ce livre alterne « des photographies d'actualité, d'autres relevant du chapitre des œeuvres d'art, d'autres encore qui se révèlent être de véritables photos-textes (photos-opinions, photos-symboles) ", pour qu'un jour, « à la mort du dernier protagoniste de cette étonnante aventure, nous soyons nombreux à lire, finalement, dans ces photographies, le récit véridique de la façon dont nous avons muselé quarante-huit années de misère ${ }^{16}$ ". Opérant des coupes successives et des changements d'échelle, le récit photographique offre une grammaire de la révolution, oscillant entre la condition du changement et le potentiel d'anticipation qui lui est inhérent. Il œuvre pour l'inscription d'une mémoire collective et

2a-b. Sérgio Guimarães (dir.), Da resistência à libertação (première de couverture et double page 52-53), Lisbonne, Mil Dias, 1977.

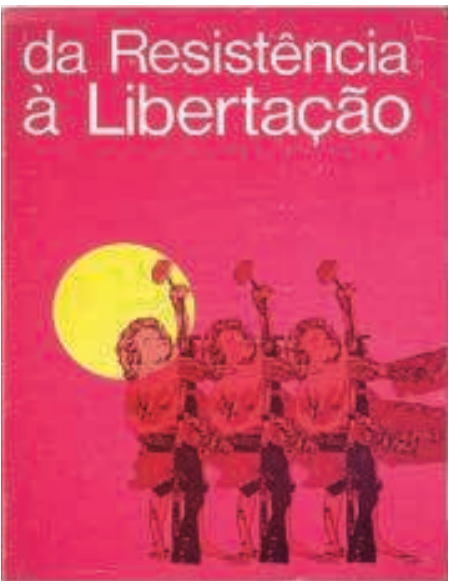
plurielle, comme l'évoque Michel Foucault lorsqu'il écrit : « On se soulève, c'est un fait; et c'est par là que la subjectivité (pas celle des grands hommes, mais celle de n'importe qui) s'introduit dans l'histoire et lui donne son souffle ${ }^{17}$."

Da resistência à libertação (1977; fig. 2a-b), publié aux éditions Mil Dias ${ }^{18}$, est un autre ouvrage produisant une documentation visuelle du processus révolutionnaire en cours qui témoigne de l'usage du livre pour légitimer la création de l'histoire par le biais des images. Dans un but d'intervention politique et sociale, tout au long des cent soixante-huit pages, nous est présenté un panorama d'images, depuis l'implantation de la République portugaise en 1910 jusqu'au $1^{\text {er }}$ mai 1974. Tout comme Portugal Livre, cet ouvrage est un document d'époque, aux apparences de manifeste graphique et ses auteurs considèrent, comme l'écrit Orlando Neves dans son introduction, que l'image est l'expression la plus intelligible de la mémoire. À l'instar d'autres initiatives commémoratives de cette période, tels que Portugal, um ano de revolução 1974-1975 (1975)

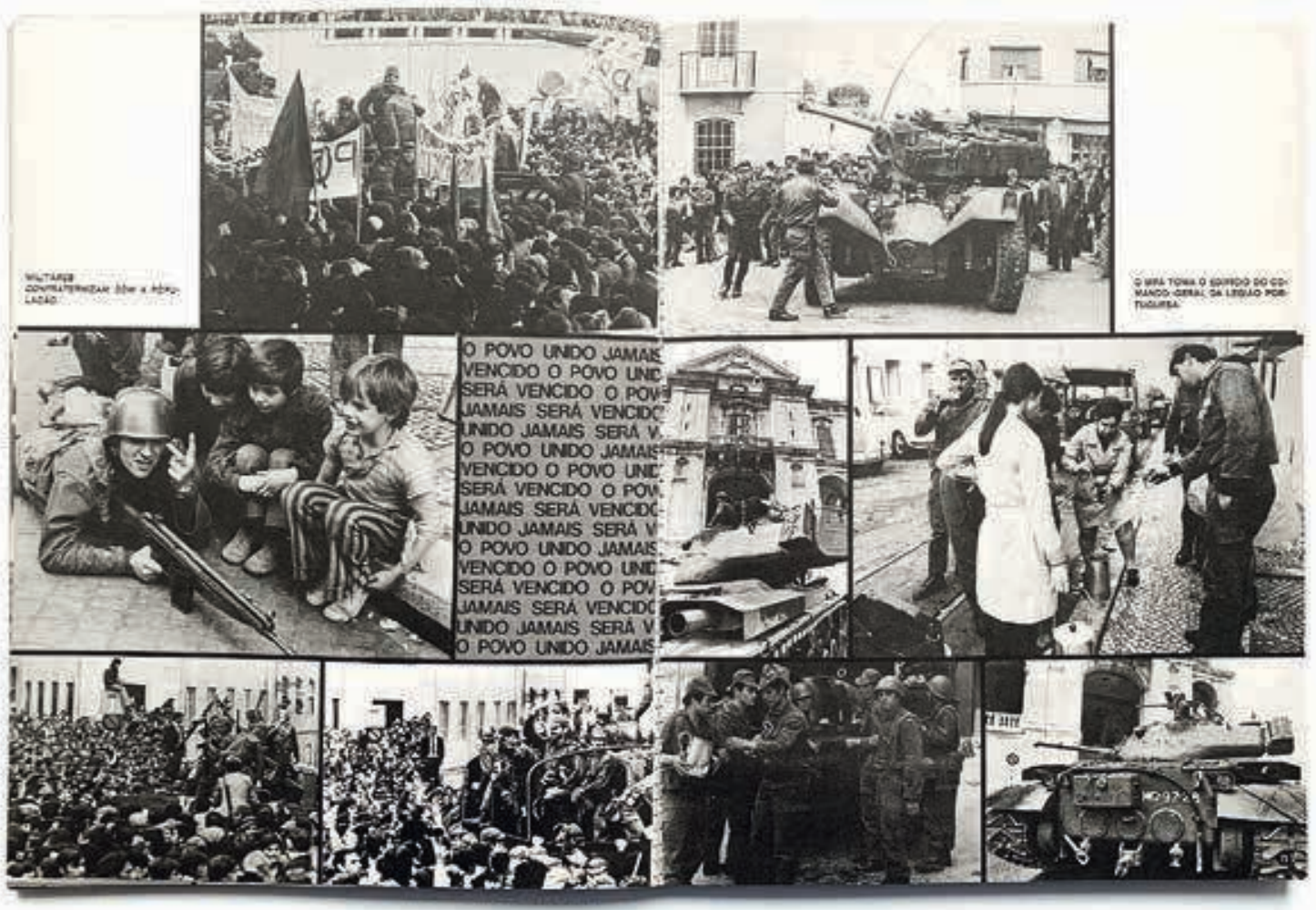



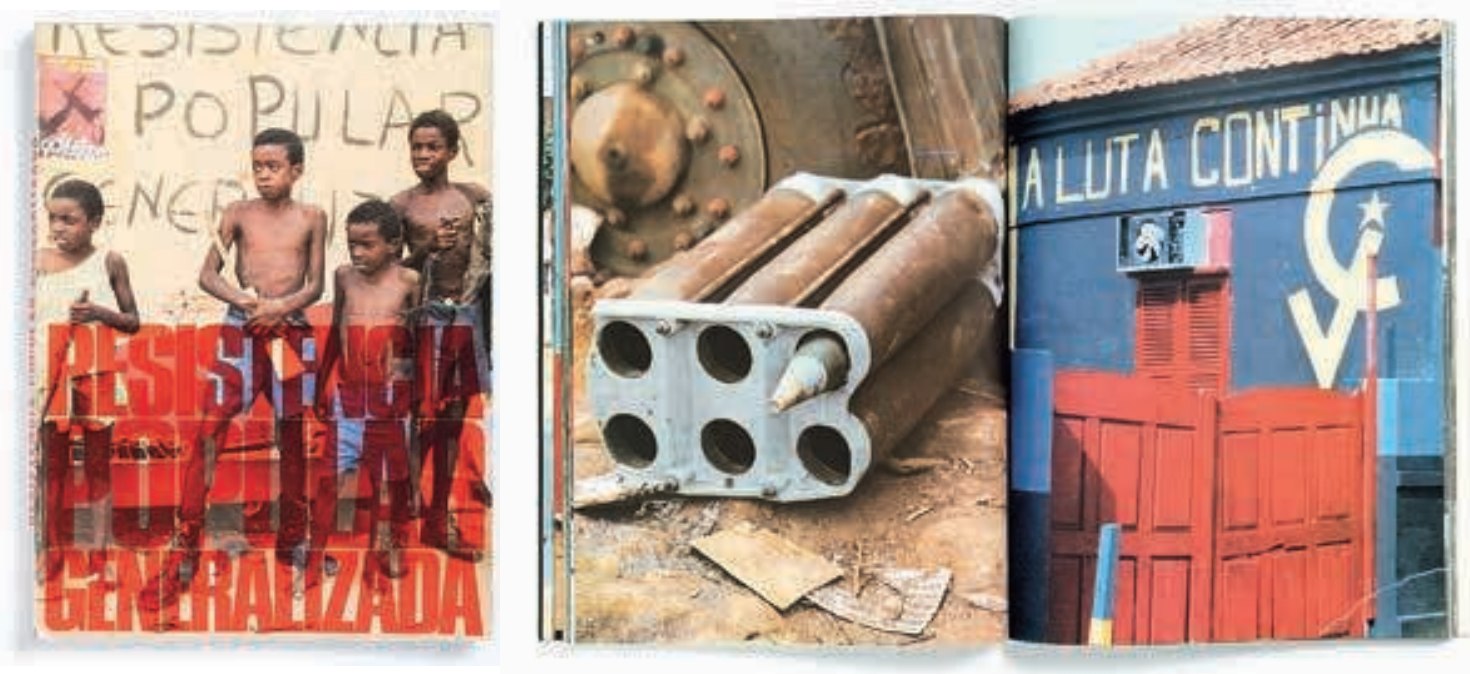

3a-b. República popular generalizada (première de couverture et double page 80-81), Louanda, Ministério da informação da república popular de Angola, 1977. ou encore Portugal Livre, cet ouvrage est édité dans le prolongement d'une exposition éponyme, présentée dans le cadre du troisième anniversaire de la révolution, entre le 25 avril et le 15 mai 1977, au Mercado do Povo, à Lisbonne. Coordonné par Orlando Neves et conçu par Sérgio Guimarães, il combine une documentation graphique et des photographies d'Abel Fonseca, Alberto

Gouveia, Alfredo Cunha, Eduardo Gageiro, Fernando Baião, Francisco Ferreira, Hernando Domingues, João Paiva, José Tavares et du Centro de intervenção para o desenvolvimento Amílcar Cabral (CIDAC, Lisbonne). Sa conception dénote une certaine expérimentation graphique, et la manière dont les images d'archives, les coupures de presse, les affiches, les slogans et les extraits de discours politiques y sont agencés évoque, de façon complexe et ironique, la poésie portugaise expérimentale des années 1960-1970. Par ailleurs, la constitution d'un index des documents en portugais, en français et en anglais et son tirage à onze mille exemplaires attestent l'ambition de diffusion et l'engagement politique certain de la maison d'édition. La photographie placée en couverture - celle d'un enfant plaçant un oeillet dans le canon d'un fusil HK G3, mise en scène en studio par Sérgio Guimarães lui-même - est du reste emblématique de la révolution des Fillets.

Contrairement à ce qui se passe dans d'autres pays européens, le processus de décolonisation au Portugal a eu lieu en pleine période révolutionnaire. Cet " effet domino ${ }^{19}$ " s'est avéré décisif et marque l'ensemble des processus d'indépendance des anciennes colonies. Comme le décrit António Costa Pinto : « La crise profonde de l'État et la forte mobilisation sociale qui a succédé au renversement du régime ont introduit une dynamique de rupture qui ne saurait se limiter à la sphère politique. Celle-ci exerce également une forte pression anticapitaliste ${ }^{20}$. " Dans le contexte angolais, cette logique de rupture acquiert une dimension plus complexe et plus violente. La guerre civile éclate suite à la proclamation de l'indépendance du 11 novembre 1975, ce qui engage une polarisation internationale et la création de différentes factions du conflit, impliquant notamment l'Union soviétique, Cuba, l'Afrique du Sud et les États-Unis. Il faut, à ce propos, établir un parallèle avec le livre Resistência popular generalizada (fig. 3a-b), publié en 1977 par le ministère de l'Information de la république populaire d'Angola dans une édition trilingue (portugais, anglais et français) - ouvrage considéré comme un outil de propagande du MPLA et des Forces armées populaires de libération de l'Angola (FAPLA). 


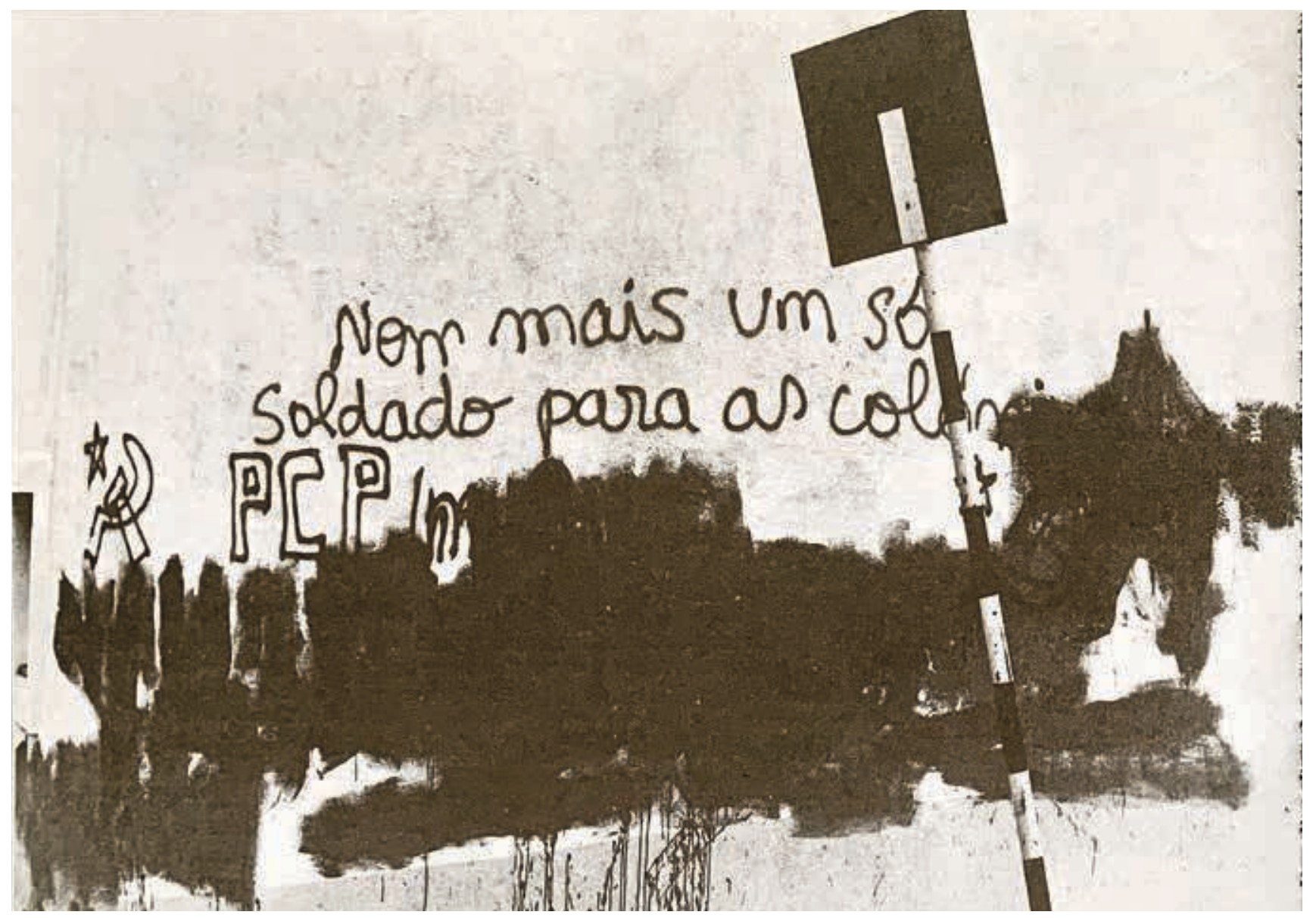

En retraçant l'histoire de la résistance et de la lutte pour l'indépendance, l'ouvrage Resistência popular generalizada, dont le titre fait référence au mouvement populaire mobilisé par le MPLA, assume l'influence politique et militaire du parti, y compris celle des organisations subalternes qui ont revendiqué la participation des femmes et des enfants dans la transformation politique et sociale de l'Angola - tels que l'Organisation des femmes angolaises (OMA) ou l'Organisation des pionniers d'Angola (OPA), et qui s'opposent à l'action de l'UNITA, présidée par Jonas Savimbi et du Front national de libération de l'Angola (FNLA). Le début du livre est constitué d'images et de textes rejetant les symboles de la présence coloniale portugaise : du démantèlement des statues des héros portugais, en passant par les traces du départ précipité des troupes portugaises, et la dénonciation et l'analyse des effets du système colonial, jusqu'à la proclamation socialiste de la nouvelle nation. Dès les premières pages, on peut lire :

Si le colonialisme naît et se développe, dans notre pays, selon une violation des institutions existantes, il provoque nécessairement un ensemble multiple de réactions - qui la plupart du temps sont aussi des réactions violentes. Le résultat dialectique de ce conflit d'intérêts, c'est l'histoire de la résistance populaire elle-même. C'est la formation, dans la lutte, d'une conscience nationale ; et la formation des moyens structurels et organisationnels que cette prise de conscience implique ${ }^{21}$.

À l'objectif de propagande s'ajoute une dimension pédagogique d'accessibilité et de massification de l'information, comme le rappellent les pages consacrées aux articles 


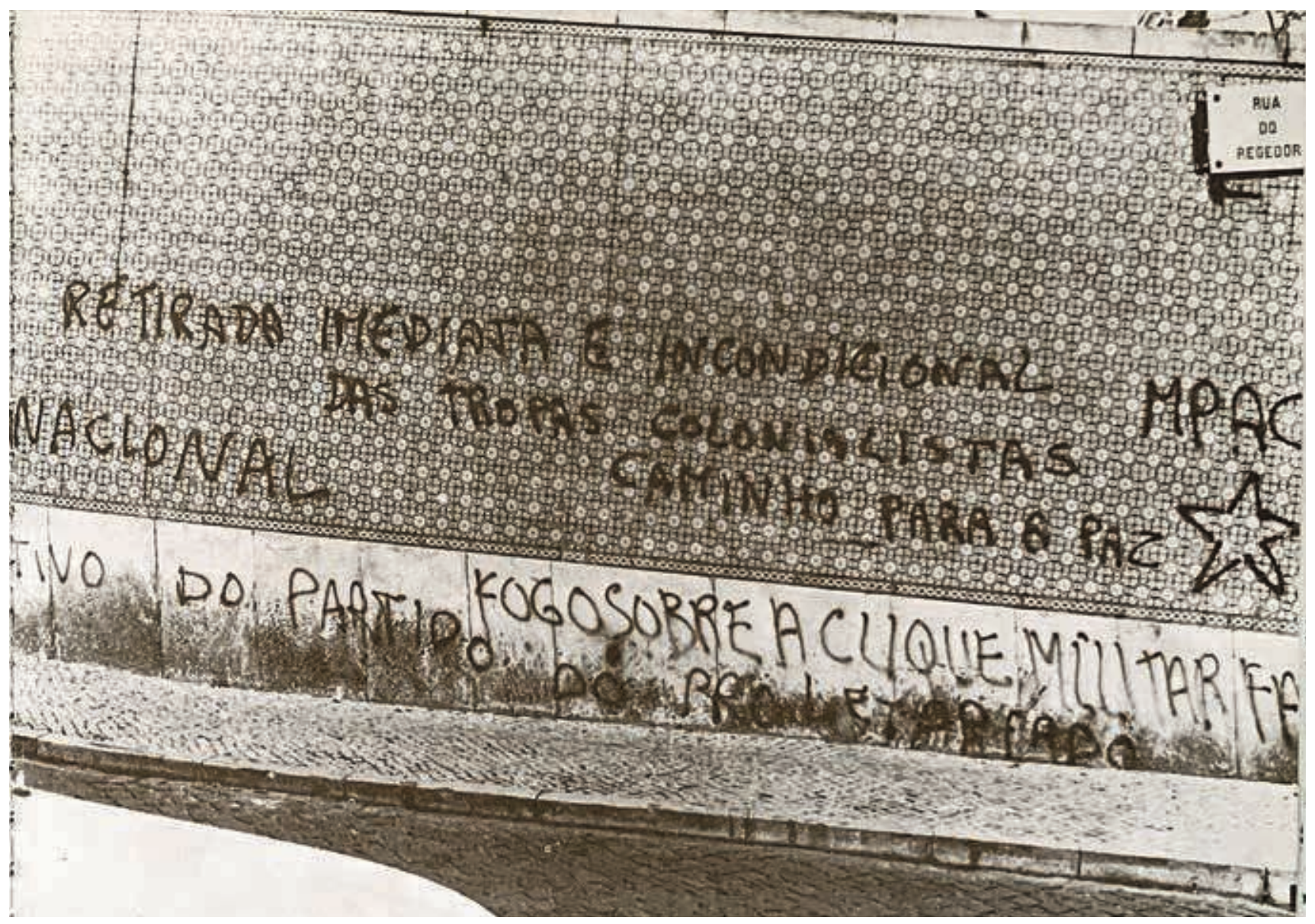

manuscrits d'un journal mural, qui nous renseigne à propos de la prise de conscience politique du peuple angolais, de la valorisation des campagnes d'alphabétisation ou encore de la formation politique et idéologique réalisées par l'OMA et les Jeunes du MPLA.

Outre sa conception graphique, l'un des aspects représentatifs du caractère exceptionnel de cet ouvrage reste la qualité des reportages photographiques, tant par leurs cadrages audacieux que par leur emploi expressif de la couleur. Il s'agit en effet d'une technologie onéreuse dont l'accès est alors limité, en raison d'un manque d'approvisionnement mais aussi d'une carence de connaissance des

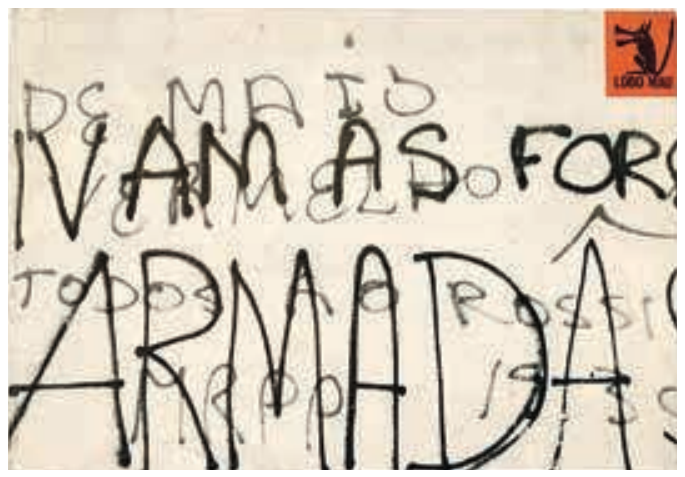

4a-b. José Marques, As Paredes em liberdade (première de couverture et double page, n.p.) Lisbonne, Editorial Teorema, 1974. techniques de développement dans l'ensemble des studios commerciaux de photographie en Angola.

Les photographies ne sont pas signées, mais certaines ont été prises par les photographes Mário António Roças et Carlos Alberto Guimarães, alors membres du département de photographie du ministère de l'Information du gouvernement provisoire de l'époque. Comme l'a souligné Carlos Alberto Guimarães, 
dans ce contexte, la pratique de la photographie était le reflet du processus de transition politique lui-même : «Un certain nombre de photographes angolais émergents durant la période pré- ou postindépendance ont été formés dans les studios portugais. Au début, ils en étaient les gardiens. Puis, ils ont gagné la confiance de leur patron. On leur a enseigné les techniques de laboratoire. Ils sont d'abord devenus imprimeurs, puis photographes ${ }^{22}$. "

En soulignant le rôle crucial des images dans le processus de décolonisation et dans les nombreuses revendications nationalistes et identitaires ${ }^{23}$ qui lui sont liés, Resistência popular generalizada présente un montage disruptif d'images. Cependant, ce dernier n'échappe pas à une double instrumentalisation : soit par la permanence des vestiges de l'occupation coloniale portugaise, assumant une logique de contre-discours ; soit par l'influence qu'elle exerce sur les manières de penser et de formuler l'identité du nouvel État-nation angolais, à travers son articulation avec le programme idéologique socialiste et les diverses actions de contreculture anti-impérialiste sur le plan international.

\section{Du Portugal au Mozambique : décolonisation des murs et liberté d'expression}

Toutes les tentatives de démocratie participative, quelle que soit leur forme, qui ont eu lieu durant le Processus révolutionnaire en cours (PREC) se sont appropriées la rue de façon spontanée et volontaire. Elles l'ont toutes revendiquée comme un espace de dissémination des mots et des images postrévolutionnaires, que ces mouvements soient populaires, associatifs, ou orientés par le Mouvement des forces armées - notamment, avec la création des " campagnes de dynamisation culturelle " pilotées par la Comissão dinamizadora central (CODICE).

Dans tout le pays, ces actions collectives ont marqué la détermination populaire d'un accès à la liberté d'expression. Grâce au placardage d'affiches, d'autocollants ou de slogans improvisés sur les murs et à la création de peintures murales plus complexes, comme celles réalisées à Lisbonne (le 10 juin 1974), à Viseu (en avril 1975), ou à Évora (les 5 et 6 juillet 1975) $)^{24}$. De nombreuses peintures murales et autres impressions graphiques de la révolution ont été sauvées grâce au cinéma et à la photographie, ce qui a permis de garantir leur visibilité et leur prévalence dans le temps. Leur publication sous forme d'ouvrages, à l'instar d'œuvres comme Les murs ont la parole (1968) du reporter Julien Besançon ; Pintadas del referendum (1977) du collectif Diorama ; ou encore The Writing on the Wall (1976) du photographe anglais Roger Perry, nous offrent une cartographie et un inventaire historique essentiels à leur examen et analyse. As Paredes em Liberdade (fig. 4a-b) est un ouvrage publié en août 1974 par la nouvelle maison d'édition Editorial Teorema ${ }^{25}$, dont la conception graphique revient à Fernando Felgueiras et Amélia Afonso. Il rejoint la collection "Lobo Mau », présentant cent quarante-huit pages de photographies prises par José Marques ${ }^{26}$, des slogans et les mots d'ordre inscrits sur les murs de Lisbonne entre mai et juin 1974. Le livre s'ouvre sur un texte court et anonyme, qui fait directement référence aux médias de presse clandestine : "reflet saisissant de la conscience politique, du non-conformisme, d'une soif de liberté au cour de l'oppression ${ }^{27}$ ".

L'appropriation de l'espace public pour la diffusion de messages contestataires et politiques provoque des revendications contradictoires et des délations réciproques, ce moyen étant utilisé par les diverses structures partisanes de gauche et de droite. La seconde partie du livre revient particulièrement sur les slogans de rejet ou d'approbation de la guerre coloniale et sur le soutien ou la dénonciation du processus de décolonisation. Certaines formules comme " Guerre coloniale = Guerre du Capital », ou " Pas un soldat de plus pour la guerre coloniale » signées par la Liga comunista internacionalista ; ou « PAIGC 


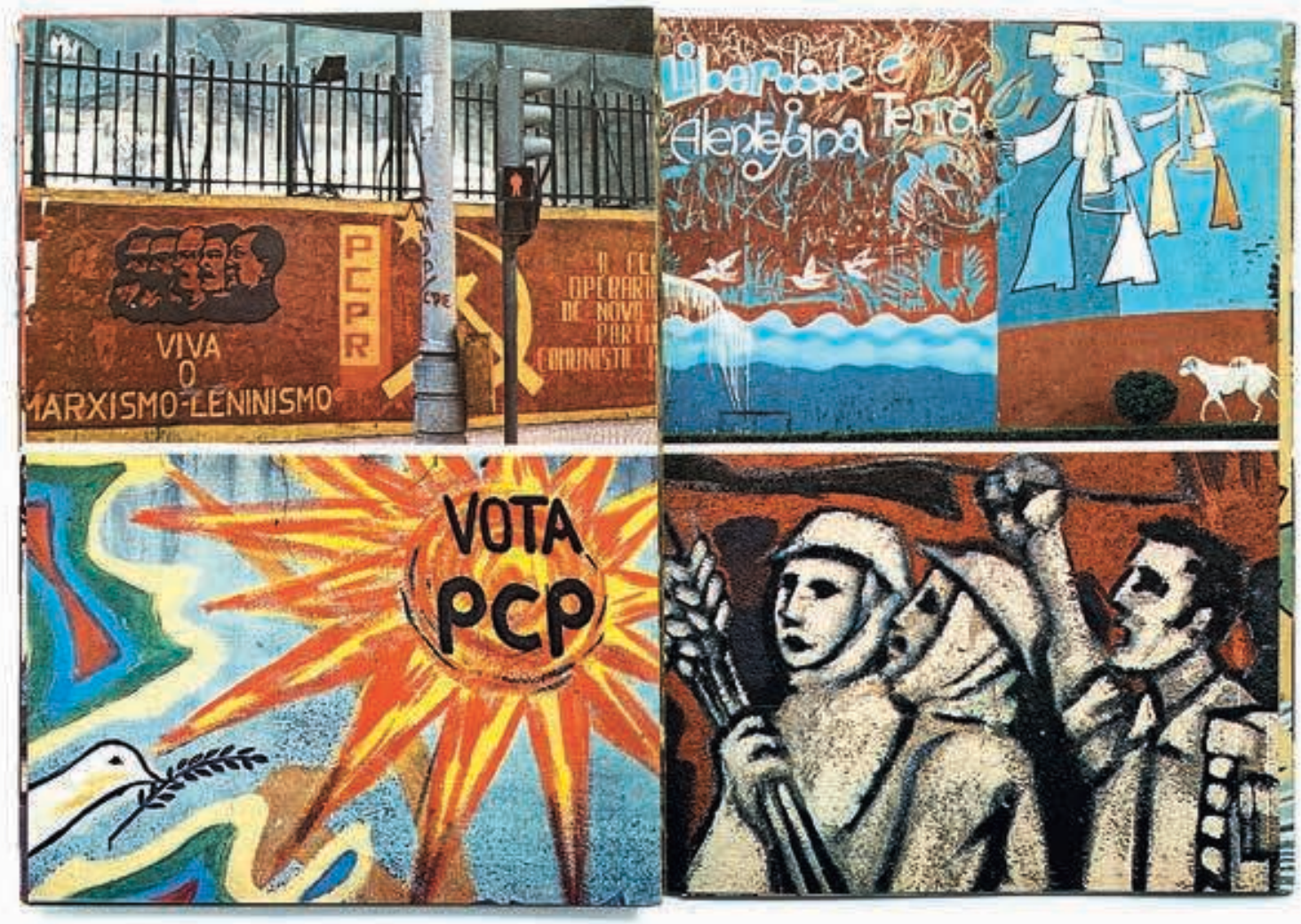

[Partido africano para a independência da Guiné e Cabo Verde] Vaincra! " par le Partido comunista português ; " Décolonisation, oui ! Cessez le feu, oui ! Dialogue, oui ! Capitulation, jamais ! " signée par le Partido popular monárquico ; ou encore «Fin de la guerre coloniale ! Retour des soldats ! " du Movimento democrático português / Comissão democrática eleitoral, sont autant d'exemples des messages contradictoires ayant permis à la société portugaise de se sensibiliser au problème de la décolonisation, sans craindre la censure.

Dans son essai de design graphique Pode-se escrever com isto, l'artiste Ernesto Melo e Castro décrit une exceptionnelle «flambée de visualisme populaire ${ }^{28}$ " qui occupe activement les rues :

Écrire sur les murs est un geste révélateur de la liberté d'un peuple. C'est une manifestation collective de la puissance de sa volonté. [...] Le Portugal s'est transformé en un immense poème visuel, qui se transformait, chaque jour, pendant deux ans, car tout le monde savait écrire et tout le monde écrivait ; car tout le monde savait lire et tout le monde lisait ${ }^{29}$.

As Paredes na revolução (fig. 5a-b), publié en 1978 à l'initiative de

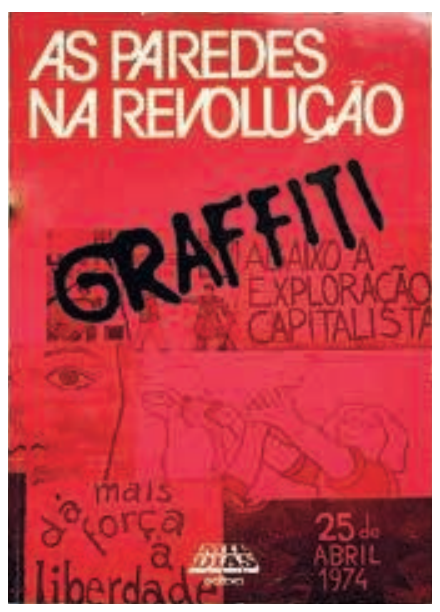

5a-b. Sérgio Guimarães (dir.), As Paredes na revolução (première de couverture et double page, n.p.), Lisbonne, Mil Dias, 1978. Sérgio Guimarães aux éditions Mil Dias, poursuit l'enregistrement de ce patrimoine éphémère. Au moyen d'une frise qui rassemble quatre photographies sur une double page, les peintures murales emblématiques et les gravures de rue se croisent pour former un inventaire inédit, sur un même plan dynamique, offrant ainsi une illustration des aspirations de la société portugaise après la révolution. Les photographies, sans signature ni légende, apparaissent dans 
le livre sans la moindre référence chronologique ou géographique. Cela permet d'élargir ce territoire d'images « qui couvrait le Portugal entier dans ces mois révolutionnaires ».

Comme l'écrit Sérgio Guimarães :

6a-b. Albie Sachs (dir.), Imagens de uma revolução (première de couverture et double page, n.p.), Maputo, Partido Frelimo, 1984.

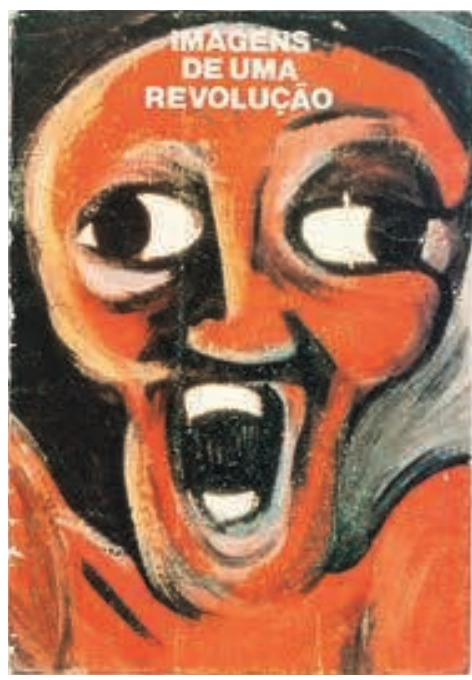

Le peuple a exprimé ce qui était considéré comme tabou et interdit. Cette soif de liberté a transposé les richesses picturales et créatives sur les murs des villes. [...] Qu'il s'agisse de simples expressions partisanes, ou qu'elles transmettent de véritables témoignages d'une région ou d'une communauté, ces inscriptions murales font partie des plus beaux événements de la révolution des Eillets ${ }^{30}$.

Cet ouvrage indique comment les peintures murales se sont transformées en outils d'intervention. Il tisse les liens de notre mémoire historique, en réaffirmant l'expression artistique dans l'espace urbain, phénomène que l'on retrouve en Angola et au Mozambique lors des scènes de liesse, après la proclamation d'indépendance.

Imagens de uma revolução (1983 ; fig. 6a-b), publié par la Zimbabwe Publishing House, est un ouvrage assez proche du précédent, qui étudie l'explosion de l'art urbain au Mozambique et rend compte des images et des slogans qui ont illuminé la ville de Maputo. L'euphorie de ces premières images postcoloniales est visible grâce à la détermination et à l'engagement du juge et activiste Albie Sachs, exilé au Mozambique suite à son implication dans la lutte

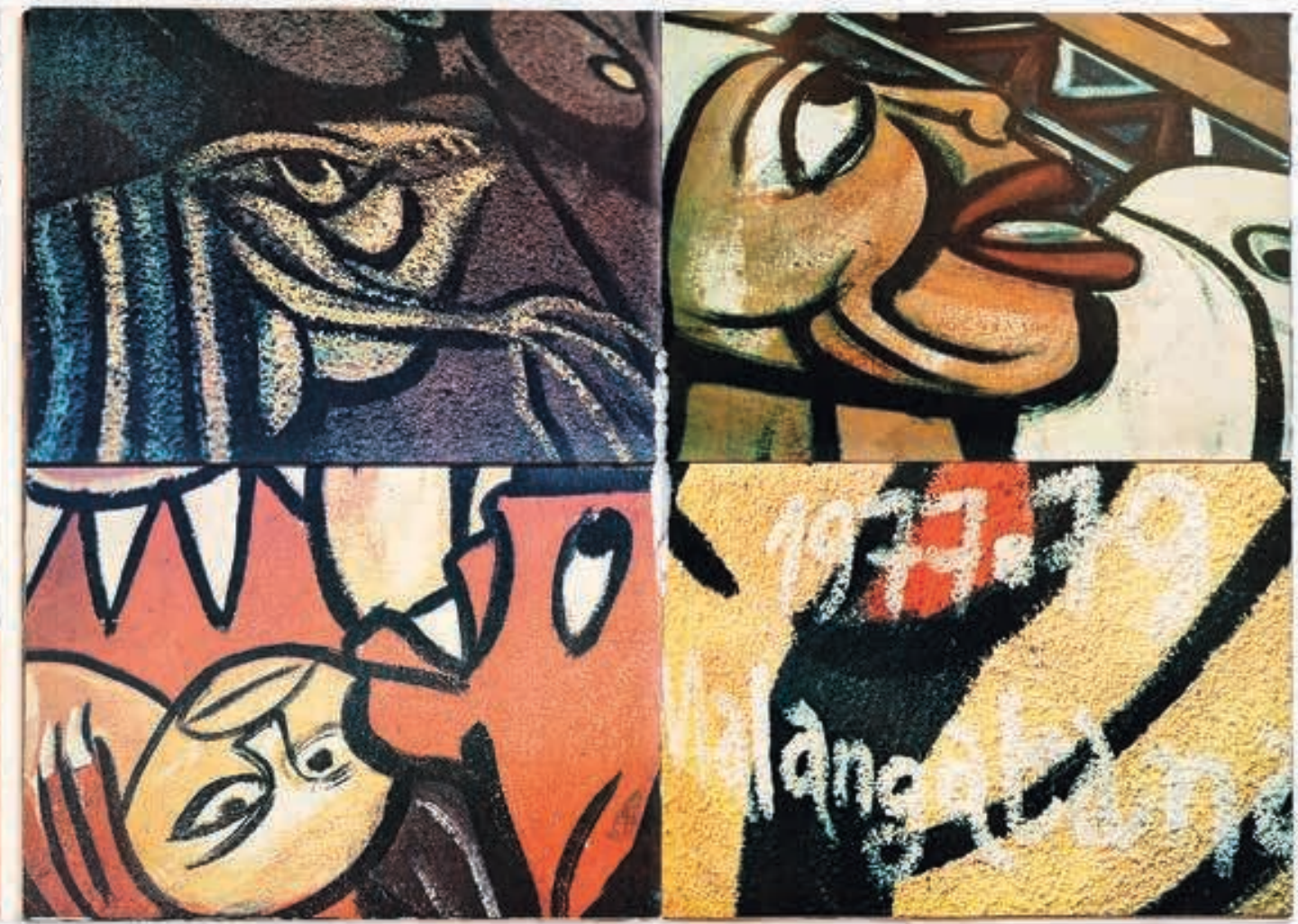


contre l'apartheid. Il a coordonné la sélection et la publication de ce vaste ensemble de photographies de peintures murales après la proclamation de l'indépendance, en collaboration avec l'écrivain et designer anglais David King. En entremêlant les vues panoramiques et les plans détaillés, cet ouvrage rend brillamment compte d'une " représentation lucide de la révolution, produite par la révolution et pour la révolution ${ }^{31}$ ".

À l'instar de As Paredes na revolução, cet ouvrage démontre le caractère tout à fait unique des peintures murales et la façon dont la spontanéité des slogans a progressivement cédé la place à un type d'intervention d'une autre envergure, à l'échelle de l'organisation collective. Des peintres tels que Malangatana Nguenha, Mankeu et João Craveirinha ont joué un rôle actif dans les mouvements de libération nationale, soutenus par des artistes exilés comme la paysagiste chilienne Moira Toha. Ensemble, ils ont permis la création des fresques de l'hôpital central de Maputo, du cinéma Matchedie, du ministère de l'Agriculture et des jardins du Museu de historia natural, au cours de la décolonisation. Selon l'analyse de Sachs, qui se réfère au parcours singulier du peintre Malagantana, il s'agit également d'un changement effectif dans l'univers pictural de ces auteurs : " La victoire est arrivée soudainement et de l'extérieur de leur espace social. Malgré la rapidité avec laquelle les colonialistes ont disparu, l'intériorisation du sens de l'indépendance a été plus lente et plus complexe ${ }^{32}$."

Contrairement à l'imagerie triomphaliste des représentations coloniales, ces peintures murales révèlent l'histoire de la lutte et de la résistance, à travers des scènes figuratives qui confondent l'expression des atrocités subies, la poursuite de la guérilla et l'hommage aux protagonistes de la lutte armée, tels qu'Eduardo Mondlane et Samora Machel. Grâce à la collaboration de photographes comme Moira Forjaz, du réalisateur et producteur Sol Carvalho, mais aussi au concours extérieur de la photographe américaine Susan Meiselas, sept peintures murales sont documentées tout au long de l'ouvrage et étudiées en détail. Ce palimpseste d'images met en évidence l'autoreprésentation de l'histoire d'un peuple qui pose un regard sur son passé, dans le but de composer et d'anticiper son avenir. Dans cette polysémie complexe, les images peuvent devenir des instruments de décolonisation de l'imaginaire colonial.

L'image de couverture retenue par Albie Sachs et David King est tirée de la fresque collective De Rovuma à Maputo, réalisée en 1977 dans le nouveau palais présidentiel. Elle nous permet d'invoquer le cri du peintre expressionniste Edward Munch : l'angoisse existentielle est ici maximisée pour représenter le fossé qui sépare la période coloniale de la période postcoloniale. Elle exprime aussi une expérience cathartique de rédemption, s'opposant à ce qui est non-dit et occulté.

Ces ouvrages publiés dans des parties du monde et des contextes politiques divers démontrent la façon dont deux forces en apparence opposées se sont reliées l'une à l'autre : d'une part, l'indépendance des territoires et des peuples colonisés et, de l'autre, la fin de la dictature qui les opprimaient. Comme l'affirme Christopher Pinney dans Photography's Other Stories (2003), ce passage en revue de l'histoire demande avant tout de combiner les formes de diffusion globale avec l'appropriation locale du médium photographique, afin de comprendre et d'approfondir les questions d'identité culturelle, au sein du système colonial, ainsi que la conscience historique qui sous-tend la nature indiciaire et descriptive de l'image photographique. Comme l'écrit Pinney dans un autre texte $^{33}$ : " Si la photographie a trop de significations pour obéir efficacement aux directives nationalistes ", il est important de reconnaître la vulnérabilité et la polyvalence des images photographiques. Il faut promouvoir « une lecture subtile des affinités entre les différentes formations discursives et les mondes imaginaires qui leur correspondent, ainsi que d'une analyse approfondie de leur pouvoir de transformation ${ }^{34}$ ». 
Ces ouvrages, paradoxalement mimétiques dans le désir de résistance et de liberté qu'ils expriment, élargissent l'univers des images postcoloniales jusqu'à un horizon de dénonciation explicite, de refus de la dictature et du modèle impérialiste, par l'affirmation d'une culture et d'une identité visuelle autonome. Si, comme l'écrivait Foucault : « Les soulèvements appartiennent à l'histoire. Mais d'une certaine façon, ils lui échappent ${ }^{35}$ ", il est encore possible de trouver, grâce à ces ouvrages, un moment où histoire, révolution et image ont progressé ensemble, en illustrant de façon paradigmatique le point de vue d'un peuple qui proclame " Je n'obéis plus. "

Traduit du portugais par Thomas Resendes. 


\section{Susana Lourenço Marques}

Susana Lourenço Marques est designer et professeure à la faculté des Beaux-Arts de I'Universidade do Porto. Titulaire d'une thèse de doctorat en sciences de la communication (Universidade Nova de Lisboa / FCSH), elle est l'auteure des ouvrages Liçoes de hospitalidade (Porto, Universidade do Porto, 2006), Pó, cinza e Nevoeiro, ensaio sobre a ausência a partir de imagens da colecção de fotografia da Muralha (Guimarães, A Oficina / CIPRL, 2018) et Ether, vale tudo menos tirar olhos: um laboratório de fotografia e história (Porto, Dafne, 2018). En 2014, elle a cofondé la maison d'édition Pierrot le Fou [URL : pierrotlefou.pt].

\section{NOTES}

1. Jean-Luc Godard, Comment ça va?, 1978.

2. Roland Barthes, La chambre claire. Notes sur la photographie, Paris, Gallimard, 1980.

3. Marc Ferro, Cinéma et Histoire, Paris, Gallimard, 1993, p. 26.

4. Jean-Luc Godard, Jean-Pierre Gorin, Anne-Marie Miéville, Ici et ailleurs, 1976.

5. Gilles Deleuze, L'Image-temps. Cinéma 2, Paris, Éditions de Minuit, 1985.

6. António Ferro, "A Política do Espírito », dans Diário de Noticias, 21 novembre 1932, p. 1. Voir Margarida Acciaiuoli, António Ferro, A vertigem da palavra. Retórica, Política e propaganda no Estado novo, Lisbonne, Bizâncio, 2013.

7. À ce sujet, voir Susana Lourenço Marques, «Imagens fugazes e triunfais. As fotografias e os filmes da primeira viagem presidencial às colónias (1938-1939): uma leitura comparativa ", dans Filomena Serra, Paula André, Sofia Leal Rodrigues (dir.), Projectos editoriais e propaganda, imagens e contra-imagens no Estado novo, Lisbonne, ICS, 2020, p. 97-115.

8. « $[\mathrm{A}]$ Nação continua no Ultramar a exercer a sua actividade, sob princípios que se integram na sua tradição humanitária e colonizadora ». Catálogo da exposição histórica da ocupação, Lisbonne, Agência geral das Colónias, 1937, vol. 1, p. XIX.

9. À l'initiative du ministre des Colonies Francisco Vieira Machado, la Missão cinegráfica às colónias de África a duré huit mois. Selon les observations de Maria do Carmo Piçarra, son objectif principal était « la production de divers documentaires sur les territoires visités, parrainés par l'Agência geral das colónias, outre la collecte d'images destinées au long-métrage de propagande Feitiço do Império [a produção, patrocinada pela Agência Geral das Colónias, de vários documentários sobre os territórios visitados, além da recolha de imagens para a longa-metragem de ficção propagandística Feitiço do Império]". Maria do Carmo Piçarra, Salazar vai ao cinema: o Jornal Português de actualidades filmadas, Coimbra, Minerva Coimbra, 2006, p. 93.
10. Eduardo Lourenço, « Retrato póstumo do nosso colonialismo inocente II ", dans Elsa Peralta, Bruno Góis, Joana Oliveira (dir.), Retornar, traços de memória do fim do império, Lisbonne, Edições 70, 2017, p. 327-341.

11. "On the African continent, this practice of cultural decolonization - based on the seminal writings of thinkers such as Frantz Fanon or Amilcar Cabral manifested in visual forms during periods following independences as part of the process to conceptualize national culture and political agitation. " Nadine Siegert, "Another World is Possible": The Socialist Mural as Visual Anticipation of a New Society ", dans Ute Fendler, Katharina Fink et al. (dir.), Revolution 3.0. Iconographies of Radical Change, Munich, AVM Edition, 2019, p. 74-103, ici p. 76.

12. " [S]ervir um rol de novos fins, incluindo a construção de uma persona, novas pertenças de classe e nacionalismos pós-coloniais ». James J. Ryan, « Introdução. Fotografia colonial », dans Filipa L. Vicente (dir.), O Império da visão: fotografia no contexto colonial português (1860-1960), Lisbonne, Edições 70, 2014, p. 31-42, ici p. 40.

13. " $[\mathrm{O}]$ velho imaginário da "revolução" e do "anticolonialismo", a velha temática antiimperialista, com as teses nativistas ». Achille Mbembe, "As formas africanas de auto-inscrição », Estudos afro-asiáticos, ano 23, no 1, 2001, p. 171-209, ici p. 191 [DOI : 10.1590/S0101546X2001000100007].

14. " [Q]uarenta e oito das quais podemos caracterizar como editoras da revolução, por terem como factor principal do seu surgimento e da sua actuação o processo revolucionário iniciado em 1974 ». Flamarion Silva, « Livros que tomam partido: a edição política em Portugal, 1968-80 ", thèse de doctorat, Universidade de São Paulo, Faculdade de Filosofia, Letras e Ciências Humanas, 2013, p. 45.

15. " Portugal continua a imaginar-se enquanto império, imaginação essa na qual a guerra e o "retorno" continuam sem lugar de inscrição. » Elsa Peralta, Joana Oliveira, "Pós-memória como herança: fotografia e testemunho do "retorno" de África ", dans Manuel Carlos Silva, Sheila Khan, Francisco Azevedo Mendes (dir.), Configurações, Revista de sociologia, no 17, 2016, p. 181-197, ici p. 187 [DOI : 10.4000/configuracoes.3290].

16. « [F]otografias de actualidade, outras englobáveis no capítulo de obra de arte, outras ainda que são autênticas fotografias-textos (fotografias-opiniões, fotografias símbolos) [realizadas para que] um dia, quando já não subsistir nenhum protagonista da aventura espantosa, muitos de nós todos, enfim, lerão nestas fotografias o relato verídico de como açaimámos 48 anos de miséria ». Fernando Assis Pacheco, "Grandola, vila morena terra da fraternidade, o povo é quem mais ordena, dentro de ti, ó cidade... ", dans Portugal Livre, cat. exp. (Lisbonne, Palácio Foz, 1974), Lisbonne, Editorial O Século, 1974, p. 5.

17. Michel Foucault, "Inutile de se soulever? » (Le Monde, 1979), dans Dits et écrits : 1976-1979, Paris, Gallimard, t. III, 1994, p. 791-793, ici p. 793.

18. Fondée par le photographe et publiciste Sérgio Guimarães, Mil Dias est une maison d'édition qui n'a publié que quatre livres consacrés au PREC, notamment 
Da resistência à libertação (1977), Diário de uma revolução (1978), As Paredes na revolução (1978) et O 25 de Abril visto pelas crianças (1978).

19. Norrie MacQueen, The Decolonization of Portuguese Africa: Metropolitan Revolution and the Dissolution of Empire, Londres, Longman, 1997.

20. « $[\mathrm{A}]$ profunda crise do Estado e a forte mobilização social que se seguiu ao derrube do regime, introduziu uma dinâmica de ruptura que não se limitou à esfera politica, incluindo uma forte pressão anticapitalista. » António Costa Pinto, O fim do império português, a cena internacional, a guerra colonial, e a descolonização, 1961-1975, Lisbonne, Livros Horizonte, 2001, p. 65.

21. «Se o colonialismo surge e se desenvolve, no nosso país, como uma violentação das estruturas existentes ele provoca necessariamente um conjunto multiforme de reacções - muitas vezes também violentas - o produto dialético deste choque de interesses é a história, ela mesma, da resistência popular, é a formação, na luta, da consciência nacional e das formas estruturais e organizativas que essa tomada de consciência implica. » República popular generalizada, Louanda, Ministério da Informação da República popular de Angola, 1977, p. 4-5.

22. « [U]ma boa parte dos fotógrafos angolanos que surgiram no período antes e pós-Independência, aprenderam em estúdios de portugueses, onde começaram como guardas, e depois de ganhar a confiança dos patrões, eram-lhes ensinadas as técnicas de laboratório, tornando-se mais tarde impressores e depois fotógrafos. »Carlos Guimarães, "O processo da fotografia comercial em Angola ", Jornal de Angola, 19 août 2020 [URL : jornaldeangola.ao/ao/noticias/o-processoda-fotografia-comercial-em-angola/].

23. À ce sujet, voir : Anderson Ribeiro Oliva, «Espelhos pós-coloniais, imagens coloniais: discursos e representações sobre os portugueses em livros didáticos angolanos de História elaborados no pós-independência (1979-2000) », dans José Macedo (dir.), Anos 90 Revista do programa de pós-graduação em história, vol. 21, n 40, décembre 2014, p. 227-249.

24. Un des tous premiers exemples de cette explosion visuelle est la fresque 48 artistas - 48 anos de fascismo, réalisée le 10 juin 1974 et détruite par l'incendie de 1981. Elle fut captée par le film Pintura Colectiva (1974) de Manuel Costa e Silva. Créée en un jour par un groupe de quarante-huit artistes du Movimento democrático de artistas plásticos - parmi lesquels figuraient Júlio Pomar, João Abel Manta, Nikias Skapinakis, Vespeira, Costa Pinheiro, Lourdes Castro, Ângelo de Sousa, Carlos Calvet, Victor Palla, Fernando Azevedo ou Eduardo Batarda - cette fresque collective occupait le mur de vingt-quatre mètres de haut de la Galeria de Belém. Le film Paredes pintadas da revolução portuguesa (1976) d'António Campos, écrit par António Domingues et dont la musique est composée par Fernando Lopes Graça, recueille le même type d'images de peintures murales élaborées par la Célula dos artistas plásticos du Partido Comunista Português. À ce sujet, voir Helena Elias, Catarina Valente, " Da utopia à distopia. O mural como ferramenta participativa nos espaços públicos da cidade », dans Cristina Pratas Cruzeiro (dir.), Convocarte - Revista de ciências da arte, no 5, mars 2018, p. 81-98.
25. La maison Editorial Teorema est fondée em 1973 par Carlos Araújo, rejoint en 1985 par Carlos da Veiga Ferreira.

26. Photographe de la scène théâtrale, l'œuvre de José Marques a été acquise en 2013 par le Teatro d. Maria II et exposée en 2020 sous la direction de Cláudia Madeira, Filipe Figueriedo et Teresa Flores.

27. José Marques, Paredes em liberdade, Lisbonne, Editorial Teorema, 1974, p. 3.

28. "[S] urto de visualismo popular». Ernesto M. Melo e Castro, "Pode-se escrever com isto », Colóquio Artes, no 32 , avril 1977, p. 49-50.

29. "A escrita nas paredes é um facto altamente revelador da liberdade de um povo e uma manifestação colectiva da força comunicativa da sua vontade. [...] Assim Portugal se transformou num enorme poema visual que todos os dias, durante dois anos, se transformou, porque todos podiam escrever, e escreviam; porque todos sabiam ler e liam. » Ibidem.

30. "O povo aí expressou o que lhe era secreto e proibido. E, deste modo, a riqueza da criação, quer temática quer pictural foi transporta para as paredes, numa ânsia de libertar a liberdade [...] as inscrições de parede, quer elas sejam meros reflexos partidários, quer contem histórias individuais de uma região ou um povo, constituem dos mais belos momentos do grande surto de liberdade que o 25 de Abril proporcionou. " Sérgio Guimarães, As Paredes na revolução, Lisbonne, Mil Dias, 1978, p. 3.

31. " [U]ma imagem autoconsciente da revolução, produzida pela revolução para servir a revolução. " Albie Sachs, Images of a Revolution - Mural Art in Mozambique, Harare, Zimbabwe Publishing House, 1983, p. 21.

32. "A vitória veio repentina e do exterior do seu espaço social e não obstante a rapidez com que os colonialistas desapareceram, a interiorização do significado da Independência foi mais lenta e complexa. »Ibidem, p. 17.

33. "Photography has too many meanings for any efficacious nationalist instruction. " Christopher Pinney, "Photography as Cure», dans The Coming of Photography in India, Londres, The British Library, 2008, p. 1-49, ici p. 49 (en italique dans le texte).

34. "Instead, we need a more nuanced reading of the affinities between particular discursive formations and the image worlds that parallel them, as well as sophisticated analysis of their transformational potentialities. " Christopher Pinney, "Introduction. "How the Other Half..." ", dans idem, Nicolas Peterson (dir.), Photography's Other Histories, Durham, Duke University Press, 2003, p. 1-14, ici p. 3.

35. Foucault, (1979) 1994, cité n. 17, p. 791. 